\title{
Transcription factor Kaiso regulates cell division in the developing mouse brain
}

\author{
Nina Illarionova \\ ICG SB RAS, Novosibirsk, Russia \\ nina.illarionova@gmail.com \\ Daria Fursenko \\ Institute of Gene Biology RAS, \\ Moscow, Russia
}

\author{
Maria Borisova \\ ICG SB RAS, Novosibirsk, Russia \\ zolotykh@bionet.nsc.ru \\ Daria Zabelina \\ NSU, Novosibirsk, Russia \\ dazabelina@gmail.com \\ Alexander Kulikov \\ ICG SB RAS, Novosibirsk, Russia \\ v_kulikov@bionet.nsc.ru
}

\author{
Ekaterina Bazhenova \\ ICG SB RAS, Novosibirsk, Russia \\ ekaterina.yu.bazhenova@gmail.com \\ Nikita Khotskin \\ ICG SB RAS, Novosibirsk, Russia \\ khotskin@bionet.nsc.ru
}

\begin{abstract}
Kaiso is a transcription factor that is known to regulate cell division in different cell types, including cancer cells. Kaiso binds kaiso specific binding site and/or methylated CpG islands on the promoter region of different target genes. Kaiso silencing may lead to an increase or a decrease in cell proliferation, which is cell specific and is probably dependent on the methylation status of Kaiso binding sites at the target promoter [1]. In various mammalian cell types Kaiso was shown to regulate cell proliferation via its target genes of cyclins E1 and D1 guarding the $\mathrm{G} 1$ to $\mathrm{S}$ phase transition in cell division [1].

Keywords - kaiso; cell division; cyclins; hippocampus
\end{abstract}

Motivation and aim

\section{Motivation}

Kaiso is widely expressed throughout the brain regions, however its regulation of cell division in brain development was not investigated.

Aim

Our aim was to study the role of the transcription factor Kaiso in cell division in the developing mouse brain.

\section{Methods}

The study was performed on littermate C57BL/6j mice of WT and Kaiso knockout (KO) genotype. Using the method of immunohistochemistry with a cell division marker bromodeoxyuridine we have calculated newborn cells within the $24 \mathrm{~h}$ period in the hippocampus, cortex, striatum and subventricular zone of the lateral ventricles on the second day after birth. Using real-time PCR we have analyzed expression profiles of associated with cell division genes ( $c-m y c, C C N E 1$ (cyclin E1) and CCND1 (cyclin D1)) in hippocampus, cortex and striatum at three different ages: embryonic day 16 (E16), postnatal day 2 (P2) and postnatal day 40 (P40).

\section{Results}

Our preliminary data showed that in the developing hippocampus (dentate gyrus region) there was a significant increase in the number of newborn cells in $\mathrm{KO}$ mice $(17 \pm 1)$ compared with the WT mice ( $12 \pm 1$ per $100 \mu \mathrm{m} 2 ; \mathrm{p}<0,001)$. The number of newborn cells co-stained with the neuron marker (NeuN) was also significantly greater in $\mathrm{KO}$ mice (6 \pm 1 per $100 \mu \mathrm{m} 2)$ compared with the WT mice $(4 \pm 1$ per 100 $\mu \mathrm{m} 2 ; \mathrm{p}<0,05)$.

In the cortex, striatum, and subventricular zone of the lateral ventricles, there were no significant differences between genotypes in the number of newborn cells.

We have performed expression profiling of genes associated with cell division regulation (c-myc, CCNE1 and $C C N D 1$ ) that were previously noted to be regulated by Kaiso in different cell types [1,2]. A significant decrease in the expression of the $c-m y c$ gene was shown in the hippocampus of $\mathrm{KO}$ mice at the age P2 compared to WT (KO: 2,7 $\pm 0,3$; WT: $4,5 \pm 0,7 ; \mathrm{p}<0,05)$. On the mRNA level neither $C C N E 1$ nor $C C N D 1$ expression was different between the genotypes in all selected brain regions and corresponding age groups.

\section{ACKNOWLEDGMENT}

Supported by the RFBR (18-04-00869 A), budget project (AAAA-A17-17072710029-7) and using the equipment of the Center for Laboratory Animal Genetic Resources Center, Federal Research Center of Cytology and Genetics SB RAS, supported by the Russian Ministry of Education and Science (RFMEFI62119X0023).

\section{REFERENCES}

[1] Pozner A. et al. (2016) Cell-specific Kaiso (ZBTB33) Regulation of Cell Cycle through Cyclin D1 and Cyclin E1. THE JOURNAL OF BIOLOGICAL CHEMISTRY. 291(47): 24538-24550.

[2] Koh D. et al. (2013) Kaiso is a key regulator of spleen germinal center formation by repressing Bcl6 expression in splenocytes. Biochemical and Biophysical Research Communications. 442: 177-182. 